\title{
Optimal Design of Adaptive Eigen-beam Beamforming in Electronic Sentinel System
}

\author{
Tielin LIU $^{\mathrm{a}}$, Cheng ZHANG ${ }^{\mathrm{b}}$, Lizhen ZHENG ${ }^{\mathrm{C}}$ \\ Department of Equipment Command and Management,Ordnance Engineering College, \\ Shijiazhuang, Hebei Province, P.R China \\ aemail: zcflysky@163.com, ${ }^{\mathrm{b} e m a i l}$ : zhangcheng_1975@163.com, ${ }^{\mathrm{C}}$ email:zysky508@163.com
}

Keywords: optimal design; Eigen-beam beamforming; WNG

\begin{abstract}
Eigen-beam beamforming technology is a kind of theory to achieve the demanding beam pattern using the linear combination of soundfield decomposition based on plane wave decomposition theory. The Eigen-beam beamforming technology is analyzed and the array optimization is reformulated as the beamforming optimization subject to multiple linear equality constraints firstly. Then the optimization problem is resolved in the orthogonal subspace of the constraint matrix and the optimal beamformer weights are achieved subject to robustness constraint. An optimal Eigen-beam beamforming algorithm is proposed using the achieved weights. The selection of the maximal order of the Eigen-beams and the convergence of the algorithm are analyzed. The correctness and validity of the proposed optimal algorithm is verified by computer simulations.
\end{abstract}

\section{Introduction}

Electronic sentinel localization system are wildly used to fulfill detection, localization, track and recognition tasks in modern information war, especially in bad battlefield environment. Traditional battlefield acoustic target localization algorithms used array in free field. Array must be located on some objects in practice, which may cause some influence to the array received signals[1]. Eigen-beam beamforming technology is a kind of theory dealing with array signal processing with baffles to achieve the demanding beam pattern using the linear combination of soundfield decomposition based on plane wave decomposition theory[2]. Spherical arrays are becoming a subject of interest as they allow three dimensional sampling of the soundfield and the beam can be steered to any 3D direction with the same beam pattern[3,4]. The next selection of array setup with baffles is uniform circular array (UCA)[5,6]. In present literature, the Eigen-beam beamforming technology are studied from different aspects[5-7]. however, the optimization design of adaptive Eigen-beam beamforming has not been studied.

This paper studies the optimization design of adaptive Eigen-beam beamforming using an array model of a circular array mount on a rigid cylinder. The Eigen-beam beamforming technology is analyzed firstly, and the array optimization is reformulated as the beamforming optimization subject to multiple linear equality constraints. The optimization problem is resolved in the orthogonal subspace of the constraint matrix to achieve the optimal beamformer weights. Then, the method using the optimal beamformer weights to synthesize the desired beam pattern is proposed. The selection of the maximal order of the Eigen-beams and the convergence of the algorithm are analyzed. Finally, computer simulations are implemented to verify the efficiency of the proposed method.

\section{Eigen-beam Beamforming}

Assume a UCA with radius $R$ mounted on a cylinder with the same radius in the horizontal plane perpendicular to the cylinder axes. Consider a unit magnitude incoming plane wave from $\varphi$ impinging on the cylinder, then the sound pressure around the cylinder can be expressed as[2] 


$$
P(\varphi)=\sum_{n=-\infty}^{\infty} j^{n}\left(J_{n}(k r)-\frac{J_{n}^{\prime}(k R)}{H_{n}^{\prime}(k R)} H_{n}(k r)\right) e^{j n \varphi}
$$

where $k$ is the wave number, $k=2 \pi f / c, f$ is the frequency and $c$ is the speed of sound. $(r, \varphi)$ is the observation point, $J_{n}(\cdot)$ is the $n$ th-order Bessel function, $H_{n}(\cdot)$ is the $n$ th-order Hankel function, $J_{n}^{\prime}(\cdot)$ and $H_{n}^{\prime}(\cdot)$ denote the derivative of the $n$ th-order Bessel function and the $n$ th-order Hankel function with respect to the argument respectively. The time dependency is omitted in the equation.

Let $r=R$ in Eq.1, then the sound pressure at the array location can be expressed

$$
P(\varphi)=\sum_{n=-\infty}^{\infty} j^{n} b_{n}(k R) e^{j n \varphi}
$$

where $b_{n}=J_{n}(k R)-\frac{J_{n}^{\prime}(k R)}{H_{n}^{\prime}(k R)} H_{n}(k R)$.

Use plane wave decomposition theory, the nth order Eigen-beam can be expressed as[3] $P_{n}=j^{n} b_{n} e^{-j n \varphi}$

The highest order of Eigen-beams satisfies $N \leq[k R][5]$.

Considering a square integrable function on the unite circular, this function can be expressed as the summation of Fourier series

$$
f(\varphi)=\sum_{n=-\infty}^{\infty} F_{n} e^{j n \varphi}
$$

The coefficients of Fourier series are defined as

$$
F_{n}=\frac{1}{2 \pi} \int_{0}^{2 \pi} f(\varphi) e^{-j n \varphi} d \varphi
$$

Sampling the pressure on the rigid cylinder using circular array, the array output can be calculated as

$$
y=\frac{1}{2 \pi} \int_{0}^{2 \pi} P(\varphi) w(\varphi) d \varphi
$$

where $w(\varphi)$ is the array weighting function. Because $P(\varphi)$ and $w(\varphi)$ are square integrable, $\left\{P(\varphi), P_{p}\right\}$ and $\left\{w(\varphi), w_{p}\right\}$ can be seen as two Fourier transform pairs and they satisfy the relations in Eq.5 and Eq.6. Then Eq.7 can be expressed as

$$
y=\frac{1}{2 \pi} \int_{0}^{2 \pi} \sum_{n=-\infty}^{\infty} P_{n} e^{j n \varphi} w(\varphi) d \varphi=\sum_{n=-\infty}^{\infty} P_{n} \frac{1}{2 \pi} \int_{0}^{2 \pi} w(\varphi) e^{j n \varphi} d \varphi=\sum_{n=-\infty}^{\infty} P_{n} w_{n}^{*}
$$

where $(*)$ denotes the complex conjugate.

Suppose the weighting function of the array beam pointing to $0^{\circ}$ is $w_{0}(\varphi)$ which can be expressed as Fourier series

$$
w_{0}(\varphi)=\sum_{n=-\infty}^{\infty} d_{n} e^{j n \varphi}
$$

where $d_{n}$ is the design coefficients of the weighting function. When $n<0, d_{n}$ satisfy $d_{n}=d_{|n|}$ 。

Different beam pattern can be achieved using different $d_{n}$ [8]. The array weights for the beam pattern pointing to the direction of $\varphi_{1}$ can be generally expressed as

$$
w(\varphi)=\sum_{n=-\infty}^{\infty} d_{n} e^{-j n \varphi_{1}} e^{j n \varphi}
$$

The coefficients of Fourier series is

$$
w_{n}=d_{n} e^{-j n \varphi_{l}}
$$

We can achieve beam pattern without frequency through proper weighting. Suppose $d_{n}{ }^{\prime}=d_{n} / b_{n}$, we can get

$$
w_{n}=d_{n}^{\prime} e^{-j n \varphi_{1}}=\frac{d_{n}}{b_{n}} e^{-j n \varphi_{1}}
$$

From Eq.4, Eq.8 and Eq.12 we can get 
$y=\sum_{n=-\infty}^{\infty} P_{n} w_{n}^{*}=\sum_{n=-\infty}^{\infty} b_{n} e^{-j n \varphi_{0}} \frac{d_{n}}{b_{n}} e^{j n \varphi_{1}}=\sum_{n=-\infty}^{\infty} d_{n} e^{j n\left(\varphi_{1}-\varphi_{0}\right)}$

Suppose the highest order of the decomposed Eigen-beams is $N$, then Eq.13 becomes

$y=\sum_{n=-N}^{N} d_{n} e^{j n\left(\varphi_{1}-\varphi_{0}\right)}$

The array beam pattern can be expressed as

$F=\sum_{n=-N}^{N} d_{n} e^{-j n\left(\varphi-\varphi_{1}\right)}$

For simplicity, set $d_{n}=1$ in the following discuss, the same as the beam points to the signal incoming direction, then Eq.15 becomes

$$
F=\sum_{n=-N}^{N} e^{-j n\left(\varphi-\varphi_{0}\right)}
$$

\section{Array Optimization with Multiple Linear Equality Constraints}

To achieve the beam pattern in Eq.16 using a circular array with $M$ elements, the beamformer can be formulated as the following linear system

$\boldsymbol{A} W=c \boldsymbol{B}$

s.t. $\boldsymbol{d}^{H} \boldsymbol{W}=1$

where $\boldsymbol{A}$ is the coefficient of the sound pressure, $\boldsymbol{W}$ is the weights in every element, $\boldsymbol{B}$ is the coefficient of beam vector pointing to $\varphi_{0} . c$ is a normalizing coefficient to satisfy the Eq.16(b), $\boldsymbol{d}$ is the complex pressure at each element position produced by a plane wave of unit magnitude in the beam direction.

$$
\begin{aligned}
\boldsymbol{A} & =\left[\boldsymbol{a}_{1} \boldsymbol{a}_{2}, \cdots, \boldsymbol{a}_{M}\right], \boldsymbol{a}_{m}=\left[b_{-N} e^{-j N \varphi_{m}}, \cdots, b_{0} e^{-j 0 \varphi_{m}}, \cdots, b_{N} e^{j N \varphi_{m}}\right]^{T}, \boldsymbol{W}=\left[w\left(\varphi_{1}\right), w\left(\varphi_{2}\right), \cdots, w\left(\varphi_{M}\right)\right]^{T}, \\
\boldsymbol{B} & =\left[e^{-j N \varphi_{0}}, \cdots, e^{-j 0 \varphi_{0}}, \cdots, e^{j N \varphi_{0}}\right]^{T}, \boldsymbol{d}=\left[d_{1}, d_{2}, \cdots, d_{M}\right]^{H}=\left[P\left(k R, \varphi_{1}\right), P\left(k R, \varphi_{2}\right), \cdots, P\left(k R, \varphi_{M}\right)\right]^{H},
\end{aligned}
$$

where $(\cdot)^{T}$ stands for the transpose, $(\cdot)^{H}$ stands for the Hermitian transpose. Eq.16(a) defines the desired beam pattern and Eq.16(b) ensures the signal passing without distortion.

Suppose Eq.16(a) has the minimum redundancy error, then we can get the priori estimate of $c$

$$
c \approx \frac{1}{\|\boldsymbol{B}\|^{2}}=\frac{1}{2 N+1}
$$

Eq.17 can be used to choose the highest order of the decomposed Eigen-beams.

Suppose Eq.16 has $K$ linear constrains, then Eq.16 can be expressed the minimal output power under multiple linear equality constraints according to the beam optimization theory in [9]

$$
\begin{array}{ll}
\min _{w} & \|\boldsymbol{A} \boldsymbol{W}-c \boldsymbol{B}\|_{2}^{2}=\min _{w} \tilde{\boldsymbol{W}}^{H} \boldsymbol{R} \tilde{\boldsymbol{W}} \\
\text { s.t. } & \boldsymbol{C}^{H} \tilde{\boldsymbol{W}}=\boldsymbol{g}
\end{array}
$$

where $C^{H}$ has $K$ linear independent row vectors and each of these row vectors corresponding to a linear constrains. One of the row vectors is $\boldsymbol{d}^{H}$ and the corresponding elements in $\boldsymbol{g}$ is 1 , that is to say, Eq.18(b) includes $\boldsymbol{d}^{H} \boldsymbol{W}=1$. The optimal solution of Eq.24 is [10]

$$
\tilde{W}=\boldsymbol{R}^{-1} \boldsymbol{C}\left(\boldsymbol{C}^{H} \boldsymbol{R}^{-1} \boldsymbol{C}\right)^{-1} \boldsymbol{g}
$$

\section{Optimization Design Algorithm}

An important measure of array performance is the white noise gain (WNG), which evaluates the robustness of the array[11]. The robustness of the designed array can be improved through adding WNG constraints. In array design, to insure array robustness generally demands

$$
\mathrm{WNG}=\frac{\left|\boldsymbol{d}^{H} \boldsymbol{W}\right|^{2}}{\boldsymbol{W}^{H} \boldsymbol{W}} \geq \delta^{2}
$$

where $\delta^{2}$ is set as-6dB generally. From Eq.16(b), Eq.20 is equivalent to

$\boldsymbol{W}^{H} \boldsymbol{W} \leq \delta^{-2}$

Adding WNG constraints to Eq.24 


$$
\begin{aligned}
& \min _{\boldsymbol{w}}\|\boldsymbol{A} \boldsymbol{W}-c \boldsymbol{B}\|_{2}^{2}=\min _{\boldsymbol{w}} \tilde{\boldsymbol{W}}^{H} \boldsymbol{R} \tilde{\boldsymbol{W}} \\
& \text { s.t. } \quad \boldsymbol{C}^{H} \tilde{\boldsymbol{W}}=\boldsymbol{g} \\
& \tilde{\boldsymbol{W}}^{H} \tilde{\boldsymbol{W}} \leq \delta^{-2}+1
\end{aligned}
$$

where $\tilde{\boldsymbol{W}}=\left[\begin{array}{ll}\boldsymbol{W}^{T} & w_{0}\end{array}\right]^{T}, \boldsymbol{R}=\left[\begin{array}{c}\boldsymbol{A}^{H} \\ \boldsymbol{c} \boldsymbol{B}^{H}\end{array}\right]\left[\begin{array}{ll}\boldsymbol{A} & \boldsymbol{c} \boldsymbol{B}\end{array}\right], \boldsymbol{C}=\left[\begin{array}{ll}\boldsymbol{d} & 0 \\ 0 & 1\end{array}\right], \boldsymbol{g}=\left[\begin{array}{ll}1 & -1\end{array}\right]^{T}, \boldsymbol{0}$ is $M \times 1$ column vector, $w_{0}$ is an additive variable with the initial value of $w_{0}=-1$ and it is changed with $\tilde{W}$ in the adaptive process.

The optimization problem in Eq.22 can be solved in the orthogonal linear subspace of constrain matrix $\boldsymbol{C}$ [9]. The weighting vector $\tilde{W}$ is decomposed as two orthogonal parts as

$$
\tilde{W}=W_{c}+\boldsymbol{V}
$$

where $\boldsymbol{W}_{c}$ is the linear combination of the column of $\boldsymbol{C}$, that to say, $\boldsymbol{W}_{c}$ is the distance mapping of $\tilde{\boldsymbol{W}}$ to $\boldsymbol{C}, \boldsymbol{V}$ is orthogonal with the row of $\boldsymbol{C}^{H}$, the same as $\boldsymbol{V}$ is the mapping of $\tilde{\boldsymbol{W}}$ to the null space of $\boldsymbol{C}^{H}$. The mapping matrixes are as followes

$$
\begin{aligned}
& \boldsymbol{P}_{c}=\boldsymbol{C}\left(\boldsymbol{C}^{H} \boldsymbol{C}\right)^{-1} \boldsymbol{C}^{H} \\
& \tilde{\boldsymbol{P}}_{c}=I-\boldsymbol{P}_{c}
\end{aligned}
$$

Then

$$
\boldsymbol{W}_{c}=\boldsymbol{P}_{c} \tilde{\boldsymbol{W}}=\boldsymbol{C}\left(\boldsymbol{C}^{H} \boldsymbol{C}\right)^{-1} \boldsymbol{C}^{H} \tilde{\boldsymbol{W}}=\boldsymbol{C}\left(\boldsymbol{C}^{H} \boldsymbol{C}\right)^{-1} \boldsymbol{g}
$$

$W_{c}$ can be seems as the LMS solution of constraint equation of Eq.22(b). At the same time, from Eq.26 we can get

$$
\boldsymbol{W}_{c}^{H} \boldsymbol{W}_{c}=\boldsymbol{g}^{H}\left(\boldsymbol{C}^{H} \boldsymbol{C}\right)^{-1} \boldsymbol{g}
$$

As $\boldsymbol{W}_{c}$ is independent with $\boldsymbol{R}$, the optimal solution problem in Eq.22 can be dealt with in the orthogonal subspace of $\boldsymbol{C}^{H}$.

As to the inequation of Eq.22(c), from Eq.23 we can get

$\boldsymbol{W}_{c}^{H} \boldsymbol{W}_{c}+\boldsymbol{V}^{H} \boldsymbol{V} \leq \delta^{-2}+1$

Considering Eq.27, we can get

$$
\boldsymbol{V}^{H} \boldsymbol{V} \leq \delta^{-2}+1-\boldsymbol{g}^{H}\left(\boldsymbol{C}^{H} \boldsymbol{C}\right)^{-1} \boldsymbol{g}=b^{2}
$$

where $b^{2}$ is the defined constraint parameter. So far, WNG constrains are transformed to the spherical constrains of $\boldsymbol{V}$.

As $\boldsymbol{R} \tilde{\boldsymbol{W}}(t)$ is the grads of the object function of Eq.22(a) in the $t$ iterative process, the update vector can be defined as

$$
\tilde{\boldsymbol{V}}(t+1)=\tilde{\boldsymbol{P}}_{c}[\boldsymbol{V}(t)-\mu \boldsymbol{R} \tilde{\boldsymbol{W}}(t)]
$$

where $\mu$ is the iterative step size. Suppose $\boldsymbol{V}(t)$ at the position of constraint boundary $\boldsymbol{V}^{H}(t) \boldsymbol{V}(t)=b^{2}$ after $t$ iterative times, then the update vector $\tilde{\boldsymbol{V}}(t+1)$ will be outside of the constraint boundary after nest iteration, that is to say, it is not satisfying the constraints in Eq.29. So, $\tilde{V}(t+1)$ needs be confined inside of the boundary. As $\tilde{V}(t+1)$ is perpendicular to the constraint boundary, $\tilde{\boldsymbol{V}}(t+1)$ can be confines through amplitude limit. Suppose

$$
\boldsymbol{V}(t)=\left\{\begin{array}{cc}
\tilde{\boldsymbol{V}}(t), & |\tilde{\boldsymbol{V}}|^{2} \leq b^{2} \\
b \frac{\tilde{V}(t)}{|\tilde{V}(t)|}, & |\tilde{\boldsymbol{V}}|^{2}>b^{2}
\end{array}\right.
$$

Then, the weighting vector iterative equation is

$$
\tilde{\boldsymbol{W}}(t+1)=\boldsymbol{W}_{c}+\left\{\begin{array}{cl}
\tilde{\boldsymbol{V}}(t+1), & |\tilde{\boldsymbol{V}}|^{2} \leq b^{2} \\
b \frac{\tilde{\boldsymbol{V}}(t+1)}{|\tilde{\boldsymbol{V}}(t+1)|}, & |\tilde{\boldsymbol{V}}|^{2}>b^{2}
\end{array}\right.
$$

The initial weighting vector value is set as the optimal solution without WNG constrains, the same as Eq.19. Then

$$
\begin{aligned}
& \tilde{\boldsymbol{W}}(0)=\boldsymbol{R}^{-1} \boldsymbol{C}\left(\boldsymbol{C}^{H} \boldsymbol{R}^{-1} \boldsymbol{C}\right)^{-1} \boldsymbol{g} \\
& \boldsymbol{V}(0)=\tilde{\boldsymbol{W}}(0)-\boldsymbol{W}_{c}
\end{aligned}
$$


If the optimal solution of Eq.32 satisfies WNG constraint, the iteration will finished with this solution, otherwise, it will start the constrained optimization process automatically. As in each iterative step, the constraints in Eq.20 and Eq.21 are satisfied exactly, so the round-off errors don't accumulate in the iterative process.

\section{Simulation Analysis}

Assume there is a rigid cylinder scatterer with radius $R=0.085$, and a uniform circular microphone array with 16 elements and the same radius is located around it. One impinging plane wave with frequency of $855 \mathrm{~Hz}$ comes from the direction of $30^{\circ}$, which corresponding to $k R=1.343$.

If there is no array layout distortion, the optimal beam pattern under the conditions of no WNG constrains is showed in Fig.2 and Fig3 respectively when the highest order of Eigen-beams is selected as $N=4$ and $N=5$, where the WNG is $-18.33 \mathrm{~dB}$ and $-44.39 \mathrm{~dB}$ respectively through calculating. It is indicated that the robustness can't be satisfied in this case and the higher the order of the Eigen-beams, the lower performance of robustness.

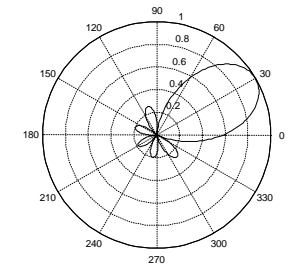

Fig.2 The optimal beam pattern without WNG constrains

$$
N=4 \text {, WNG }=-18.33 \mathrm{~dB}
$$

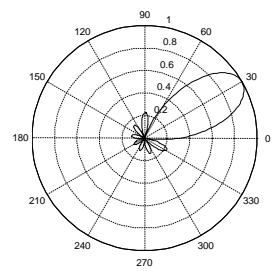

Fig.3 The optimal beam pattern without WNG constrains

$$
N=5 \text {, WNG }=-44.39 \mathrm{~dB}
$$

If there is array distortion, the array performance will be declined further. Suppose there is $3^{\circ}$ between the incoming directions of the desired signal with the actual directions. At this case WNG is $-21.76 \mathrm{~dB}$ and $-65.11 \mathrm{~dB}$ respectively corresponding to $N=4$ and $N=5$. From the analysis of Fig. 1 we can see that the highest order of Eigen-beam is $N=4$ when satisfying WNG constrains. The optimal beam pattern using the algorithm proposed in this paper is showed in Fig.4 with array distortion, in which the highest order of Eigen-beams is selected as $N=4$ and WNG $=-6 \mathrm{~dB}$. It can be seen that the achieved beam pattern using the algorithm proposed in this paper is consistent with the desired beam pattern under robustness constrains. The relationship of the object function of the proposed algorithm with iteration numbers is showed in Fig.5. It can be seen in that the proposed algorithm has fast convergence speed.

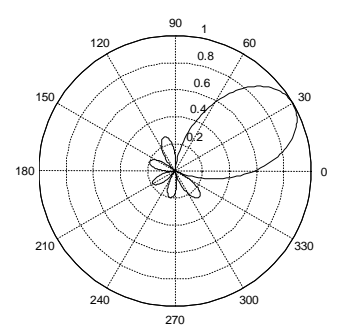

Fig.4 The optimal beam pattern with array distortion using the proposed algorithm $N=4$, WNG $=-6 \mathrm{~dB}$

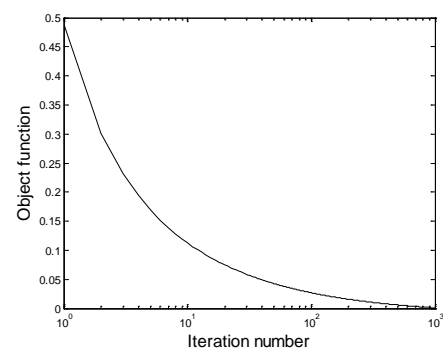

Fig.5 The relationship of object function of the proposed algorithm with iteration numbers

\section{Conclusion}

Battlefield acoustic target localization plays a great role in information war era, which is an important activity in intelligence, surveillance and reconnaissance. It has great significance for considering the effect of array baffles when head-mounted electronic sentinel systems are designed. The optimization design of adaptive Eigen-beam beamforming in sentinel system using an array 
model of a circular array mount on a rigid cylinder are studied in this paper. The array optimization is reformulated as the beamforming optimization subject to multiple linear equality constraints and the optimization problem is resolved in the orthogonal subspace of the constraint matrix to achieve the optimal beamformer weights. The correctness and efficiency of the proposed method are verified by computer simulations, which provide theoretical foundations for engineering application of head-mounted acoustic sources localization in electric sentinel systems.

\section{Acknowledgement}

In this paper, the research was sponsored by the Nature Science Foundation of Hebei Province (Project No. F2013506015).

\section{References}

[1] Tribikram K. Acoustic source localization[J]. Ultrasonics, 2014 54(1) 25-38.

[2] Teutsch H. Modal Array Signal Processing: Principles and Applications of Acoustic Wavefield Decomposition[M]. New York: Springer-Verlag, 2007.

[3] Rafaely B. Plane-wave decomposition of the sound field on a sphere by spherical convolution[J]. Journal Acoustics Society of America, 2004 116(4) 2149-2157.

[4] Rafaely B. Analysis and design of spherical microphone arrays[J]. IEEE Transactions on Speech Audio Processing, 2005 13(1) 135-143.

[5] Teutsch H, Kellermann W. Acoustic source detection and localization based on wavefield decomposition using cicular microphone arrays[J]. Journal Acoustics Society of America, 2006 120(5) 2724-2736.

[6] Meyer J. Beamforming for a circular microphone array mounted on spherically shaped objects[J]. Journal Acoustics Society of America, 2001 109(1) 185-193.

[7] Zhang C. and so on. Performance analysis of beamforming for circular array with rigid cylindrical baffle[J]. ACTA ACUSTICA, 2010 35(1) 68-75.

[8] Rafaely B. Phase-mode versus delay-and-sum spherical microphone array processing[J]. IEEE signal processing Letters, 2005 12(10) 713-716.

[9] Frost O. L. An algorithm for linearly constrained adaptive array processing [C]. Proceedings of the IEEE, 1972 60(8) 926-935.

[10]Cox H, Zeskind R M, Owen M M. Robust adaptive beamforming[J]. IEEE Transactions on Acoustics, Speech, and Signal Processing, 1987 35(10) 1365-1376.

[11] Harry L V T. Optimum array processing: part IV of detection, estimation, and modulation theory[M]. New York: Wiley-Interscience, 2002. 